PROCEEDINGS OF THE

AMERICAN MATHEMATICAL SOCIETY

Volume 139, Number 11, November 2011, Pages 3985-3997

S 0002-9939(2011)10803-7

Article electronically published on March 17, 2011

\title{
FACTOR MAP, DIAMOND AND DENSITY OF PRESSURE FUNCTIONS
}

\author{
JUNG-CHAO BAN AND CHIH-HUNG CHANG
}

(Communicated by Yingfei Yi)

\begin{abstract}
Letting $\pi: X \rightarrow Y$ be a one-block factor map and $\Phi$ be an almostadditive potential function on $X$, we prove that if $\pi$ has diamond, then the pressure $P(X, \Phi)$ is strictly larger than $P(Y, \pi \Phi)$. Furthermore, if we define the ratio $\rho(\Phi)=P(X, \Phi) / P(Y, \pi \Phi)$, then $\rho(\Phi)>1$ and it can be proved that there exists a family of pairs $\left\{\left(\pi_{i}, X_{i}\right)\right\}_{i=1}^{k}$ such that $\pi_{i}: X_{i} \rightarrow Y$ is a factor map between $X_{i}$ and $Y, X_{i} \subseteq X$ is a subshift of finite type such that $\rho\left(\pi_{i},\left.\Phi\right|_{X_{i}}\right)$ (the ratio of the pressure function for $P\left(X_{i},\left.\Phi\right|_{X_{i}}\right)$ and $P(Y, \pi \Phi)$ ) is dense in $[1, \rho(\Phi)]$. This extends the result of Quas and Trow for the entropy case.
\end{abstract}

\section{INTRODUCTION}

The present paper is devoted to studying the topic that for a given one-block factor map, how the existence of diamond and different kinds of potential functions affect the pressure function, and what is the density of the pressure. This is mainly motivated by the related works concerning entropy [2 and the dense entropy property [3]. Before formulating our results, we give some notation and background first. Let $\pi: X \rightarrow Y$ be a 1-block factor map between two one-dimensional mixing subshifts of finite type $X$ and $Y$. Then the following result is well-known:

Theorem 1.1 (Theorem 4.1.7 of [4]). Suppose $\pi: X \rightarrow Y$ is a one-block factor map between mixing subshifts of finite type (SFTs for short) and that X has positive entropy. Then either

(1) $\pi: X \rightarrow Y$ is uniformly bounded-to-one,

(2) $\pi$ has no diamond,

(3) $h_{\text {top }}(X)=h_{\text {top }}(Y)$

Received by the editors May 3, 2010 and, in revised form, September 19, 2010.

2010 Mathematics Subject Classification. Primary 37D35; Secondary 37B10, 37A35, 28A78.

Key words and phrases. Factor map, diamond, a-weighted thermodynamic formalism, density of pressure.

The first author is partially supported by the National Science Council, ROC (Contract No. NSC 98-2628-M-259-001), National Center for Theoretical Sciences (NCTS) and CMPT (Center for Mathematics and Theoretical Physics) in National Central University.

The second author wishes to express his gratitude to Professor Cheng-Hsiung Hsu for his valuable comments and thanks the National Central University for financial support.

(C)2011 American Mathematical Society 
or

(4) $\pi: X \rightarrow Y$ is uncountable-to-one on some points,

(5) $\pi$ has diamond,

(6) $h_{\text {top }}(X)>h_{\text {top }}(Y)$.

We remark here that Theorem 1.1 also holds for higher-dimensional SFTs (Theorem 3.6 of [2]). However, unlike the one-dimensional case, some stronger specification property is needed for the higher-dimensional case. Let $\Phi=\left(\log \phi_{n}\right)_{n=1}^{\infty}$ be a real-valued potential function on $X$, i.e., $\log \phi_{n}: X \rightarrow \mathbb{R}$ for all $n \in \mathbb{N}$. We define the push-forward potential in $Y$ by

(1.1) $\pi \Phi(y)=\left(\max _{x \in X: \pi(x)=y} \log \pi \phi_{n}(x)\right)_{n=1}^{\infty}=\left(\max _{x \in X: \pi(x)=y} \log \phi_{n} \circ \pi^{-1}(x)\right)_{n=1}^{\infty}$,

and define the pressure function on $X$ by

$$
P(X, \Phi)=\lim _{n \rightarrow \infty} \frac{1}{n} \log \sum_{I \in X_{n}} \sup _{x \in[I]} \phi_{n}(x)
$$

whenever the limit exists and $X_{n}$ stands for the collection of $n$-cylinders in $X$. It is of interest to know whether Theorem 1.1 holds for the pressure function. Precisely, we consider the following.

Problem 1.2. If $\pi: X \rightarrow Y$ is a one-block factor map with diamond between $X$ and $Y$, which potential functions $\Phi=\left(\log \phi_{n}\right)_{n=1}^{\infty}$ on $X$ make $P(X, \Phi)>P(Y, \pi \Phi)$ ?

Problem 1.3. Under the same assumption of Problem 1.2, what is the difference $P(X, \Phi)-P(Y, \pi \Phi) ?$

In this investigation, we have the following results:

Theorem A. Let $\pi: X \rightarrow Y$ be a one-block factor map between two mixing shift spaces $X$ and $Y$. Assume $\Phi=\left(\log \phi_{n}\right) \in \mathbf{C}_{a a}(X, T)$ (defined in (2.2)) and satisfies the bounded distortion property (defined in (2.4)). Then either

(1) $P(X, \Phi)>P(Y, \pi \Phi)$,

(2) $\pi$ has diamonds,

or

(3) $P(X, \Phi)=P(Y, \pi \Phi)$,

(4) $\pi$ has no diamond.

For the case $\pi$ has diamond, Theorem A shows that $P(X, \Phi)-P(Y, \pi \Phi)>0$ if and only if $\pi$ has diamond. This extends Theorem 1.1 to pressure for $\Phi \in \mathbf{C}_{a a}(X, T)$. For the difference $P(X, \Phi)-P(Y, \pi \Phi)$ of Problem 1.3. we have the following result.

Theorem B. Under the same assumption of Theorem $A$, let $\nu \in \mathcal{M}(Y, S)$ be the equilibrium measure on $Y$ with respect to the push-forward potential $\pi \Phi(y)=$ $\left(\max _{x \in X: \pi(x)=y} \log \phi_{n} \circ \pi^{-1}(x)\right)_{n=1}^{\infty}$ and $\mu \in \mathcal{M}(X, T)$ be the conditional equilibrium state of $\Phi$ with respect to $\nu$ (see (2.13) ) and Proposition 2.6). Then

$$
P(X, \Phi)-P(Y, \pi \Phi)=h_{\mu}(T)-h_{\nu}(S) .
$$

Theorem B indicates that the difference of $P(X, \Phi)-P(Y, \pi \Phi)$ equals $h_{\mu}(T)-$ $h_{\nu}(S)$, and it is useful for characterizing the positivity of $P(X, \Phi)-P(Y, \pi \Phi)$ by showing $h_{\mu}(T)>h_{\nu}(S)$ (see Theorem 3.1). 
On the other hand, for a dynamical system $(X, T)$, it is natural to ask what are the subsystems of $X$ and what are the possible values of the entropies (resp. pressure) of the subsystems of $X$. If $X$ is an $n$-dimensional SFT for $n \in \mathbb{N}$, Quas and Trow [3] show that for $\varepsilon>0$, there exists a proper subshift $\hat{X}$ of $X$ which is also an SFT with the property that

$$
h_{\text {top }}(X)-\varepsilon<h_{\text {top }}(\hat{X})<h_{\text {top }}(X) .
$$

If $\pi: X \rightarrow Y$ is a one-block factor map with diamond and $\Phi=\left(\log \phi_{n}\right)_{n=1}^{\infty} \in$ $\mathbf{C}_{a a}(X, T)$ with the bounded distortion property, we define the ratio of $P(X, \Phi)$ and $P(Y, \pi \Phi)$ by

$$
\rho(\pi, \Phi)=P(X, \Phi) / P(Y, \pi \Phi) \text { if } P(Y, \pi \Phi) \neq 0 .
$$

It follows from Theorem A that we have $\rho(\pi, \Phi)>1$. We ask the following questions:

Problem 1.4. Under the same assumptions of Theorem A, does there exist a family $\pi_{i}: X_{i} \rightarrow Y$ where $X_{i}$ is a subsystem of $X$ and $\pi_{i}=\left.\pi\right|_{X_{i}}$ is a one-block factor for all $i \in \mathbb{N}$ such that

$$
\rho\left(\pi_{i},\left.\Phi\right|_{X_{i}}\right)=P\left(X_{i},\left.\Phi\right|_{X_{i}}\right) / P(Y, \pi \Phi)
$$

is dense in $[1, \rho(\pi, \Phi)]$, where $\left.\Phi\right|_{X_{i}}$ stands for the restriction of $\Phi$ to $X_{i}$ ?

For Problem 1.4, we have the following result.

Theorem C. Under the same assumptions of Theorem A, there exists a family of pairs $\left\{\left(\pi_{i}, X_{i}\right)\right\}_{i=1}^{\infty}$ such that

(1) $X_{i}$ is a subsystem of $X, \forall i \in \mathbb{N}$;

(2) $X_{i}$ is an $S F T, \forall i \in \mathbb{N}$;

(3) $\pi_{i}: X_{i} \rightarrow Y$ is a one-block factor map for all $i \in \mathbb{N}$ such that $\rho\left(\pi_{i},\left.\Phi\right|_{X_{i}}\right) \neq 0$ are dense in $[1, \rho(\Phi)]$. That is, for $\varepsilon>0$, there exists an integer $k=k(\varepsilon)$ and a monotone decreasing sequence $\left\{P\left(X_{i},\left.\Phi\right|_{X_{i}}\right)\right\}_{i=1}^{k}$ such that

$$
P\left(X_{i},\left.\Phi\right|_{X_{i}}\right)-P\left(X_{i+1},\left.\Phi\right|_{X_{i+1}}\right)<\varepsilon,
$$

and, for all $p \in[P(Y, \pi \Phi), P(X, \Phi)]$, there exists $a 1 \leq j \leq k$ with

$$
P\left(X_{j+1},\left.\Phi\right|_{X_{j+1}}\right)<p<P\left(X_{j},\left.\Phi\right|_{X_{j}}\right) .
$$

The content of this paper is the following. In Section 2, we introduce the socalled a-weighted thermodynamic formalism developed recently by Barral and Feng [1. This tool is useful for the proofs of Theorem B and Theorem A, and we leave their proofs to Section 3 and give the proof of Theorem C in Section 4.

\section{Preliminaries and a-Weighted thermodynamic FORMalism}

For the reader's convenience we recall some definitions and known results in this section.

2.1. Sub-additive thermodynamic formalism. The following definitions and notation come from the recent works of Barral and Feng [1].

Definition 2.1. (1) We say that $\Phi=\left(\log \phi_{n}\right)_{n=1}^{\infty}$ is sub-additive on $X$ and write $\Phi \in \mathbf{C}_{s}(X, T)$ if there exists $C_{1}>0$ such that

$$
\phi_{n+m}(x) \leq C_{1} \phi_{n}(x) \phi_{m}\left(T^{n} x\right) \forall x \in X \text { and } n, m \in \mathbb{N} \text {. }
$$


(2) We say that $\Phi=\left(\log \phi_{n}\right)_{n=1}^{\infty}$ is asymptotically sub-additive on $X$ and write $\Phi \in \mathbf{C}_{a s s}(X, T)$ if for any $\varepsilon>0$ there exists a sub-additive potential $\Psi=$ $\left(\log \psi_{n}(x)\right)_{n=1}^{\infty}$ on $X$ such that

$$
\limsup _{n \rightarrow \infty} \frac{1}{n} \sup _{x \in X}\left|\log \phi_{n}(x)-\log \psi_{n}(x)\right| \leq \varepsilon .
$$

(3) $\Phi=\left(\log \phi_{n}\right)_{n=1}^{\infty}$ is called almost additive on $X$ and we write $\Phi \in \mathbf{C}_{a a}(X, T)$ if $\phi_{n}$ is positive and continuous on $X$ for all $n \in \mathbb{N}$ and there exists $C_{2}>0$ such that

$$
C_{2}^{-1} \phi_{n}(x) \phi_{m}\left(T^{n}(x)\right) \leq \phi_{n+m}(x) \leq C_{2} \phi_{n}(x) \phi_{m}\left(T^{n}(x)\right),
$$

$\forall x \in X$ and $n, m \in \mathbb{N}$.

(4) $\Phi=\left(\log \phi_{n}\right)_{n=1}^{\infty}$ is called the bounded distortion property if there exists a constant $C_{3}>0$ such that

$$
C_{3}^{-1} \phi_{n}(y) \leq \phi_{n}(x) \leq C_{3} \phi_{n}(y) \forall x, y \in I \in X_{n} .
$$

We introduce the following result of the variational principle for the asymptotic sub-additive potential $\Phi$ on $X$.

Theorem 2.2 (Feng and Huang, [9]). Let $\Phi \in \mathbf{C}_{a s s}(X, T)$ and $T: X \rightarrow X$ be a mixing continuous transformation. Then

$$
P(X, \Phi)=\sup \left\{h_{\eta}(T)+\Phi_{*}(\eta): \eta \in \mathcal{M}(X, T)\right\}
$$

where $\mathcal{M}(X, T)$ denotes the collection of $T$-invariant probability measures on $X$ endowed with the weak-star topology, $h_{\eta}(T)$ denote the measure-theoretic entropies of $\eta$ and $\Phi_{*}(\eta)$ is given by

$$
\Phi_{*}(\eta)=\lim _{n \rightarrow \infty} \frac{1}{n} \int \log \phi_{n}(x) d \eta(x) .
$$

A measure $\mu \in \mathcal{M}(X, T)$ attaining the supremum of (2.5) is called the equilibrium measure of $\Phi$. A measure $\mu \in \mathcal{M}(X, T)$ is called a Gibbs measure with respect to $\Phi$ if there exists a $Q_{1}>0$ such that

$$
Q_{1}^{-1} \leq \frac{\mu([I])}{\exp (-n P(X, \Phi)) \phi_{n}([I])} \leq Q_{1}, \forall I \in X_{n}, n \in \mathbb{N},
$$

where

$$
\phi_{n}([I])=\sup _{x \in[I]} \phi_{n}(x) .
$$

It follows from Theorem 2.2 that we can construct the variational principle for $P(X, \Phi)$ and $P(Y, \pi \Phi)$.

Proposition 2.3. Let $\Phi \in \mathbf{C}_{a s s}(X, T)$ and let $\pi \Phi$ be defined as in (1.1) on $Y$. Then:

(1) $\pi \Phi \in \mathbf{C}_{a s s}(Y, S)$.

(2) The two variational principles hold:

$$
\begin{gathered}
P(X, \Phi)=\sup \left\{h_{\eta}(S)+\Phi_{*}(\eta): \eta \in \mathcal{M}(X, T)\right\}, \\
P(Y, \pi \Phi)=\sup \left\{h_{\xi}(S)+(\pi \Phi)_{*}(\xi): \xi \in \mathcal{M}(Y, S)\right\} .
\end{gathered}
$$

Furthermore, if we assume $\Phi \in \mathbf{C}_{a a}(X, T)$ and satisfies the bounded distortion property, then

(3) $\pi \Phi \in \mathbf{C}_{a a}(Y, S)$. 
(4) There exist unique equilibrium measures $\mu \in \mathcal{M}(X, T), \nu \in \mathcal{M}(Y, S)$ attaining the supremums of (2.9) and (2.10) respectively.

(5) Both $\mu$ and $\nu$ satisfy the Gibbs property; i.e., (2.7) holds for $\mu$ and $\nu$.

2.2. a-weighted thermodynamic formalism. Let $(X, T)$ and $(Y, S)$ be mixing shift spaces. Assume $\Phi \in \mathbf{C}_{a s s}(X, T)$ and $\mathbf{a}=(a, b) \in \mathbb{R}^{2}$ so that $a>0$ and $b \geq 0$. Barral and Feng [1] introduce the a-weighted topological pressure of $\Phi$ :

$$
P^{\mathbf{a}}(X, \Phi)=\sup \left\{\Phi_{*}(\eta)+a h_{\eta}(T)+b h_{\eta \circ \pi^{-1}}(S): \eta \in \mathcal{M}(X, T)\right\} .
$$

A measure $\mu \in \mathcal{M}(X, T)$ attaining the supremum of (2.11) is called the a-weighted equilibrium state of $\Phi$. Let $\Phi=\left(\log \phi_{n}\right)_{n=1}^{\infty} \in \mathbf{C}_{a s s}(X, T)$, define a sequence $\Psi=$ $\left(\log \psi_{n}\right)_{n=1}^{\infty}$ of potentials on $Y$ by

$$
\psi_{n}(y)=\sum_{I \in X_{n}:[I] \cap \pi^{-1}(y) \neq \emptyset} \sup _{x \in[I] \cap \pi^{-1}(y)} \phi_{n}(x)^{\frac{1}{a}}, y \in Y,
$$

and set $\frac{a}{a+b} \Psi=\left(\log \left(\psi_{n}^{\frac{a}{a+b}}\right)\right)_{n=1}^{\infty}$. For $\nu \in \mathcal{M}(Y, S)$, a measure $\mu \in \mathcal{M}(X, T)$ is called a conditional equilibrium state of $\Phi$ with respect to $\nu$ if $\mu \circ \pi^{-1}=\nu$ and

$$
\Phi_{*}(\mu)+h_{\mu}(T)-h_{\nu}(S)=\sup \left\{\Phi_{*}(\eta)+h_{\eta}(T)-h_{\nu}(S): \eta \in \mathcal{M}(X, T), \eta \circ \pi^{-1}=\nu\right\} .
$$

Barral and Feng [1] developed the following results.

Theorem 2.4. Let $\mathbf{a}=(a, b) \in \mathbb{R}^{2}$ so that $a>0$ and $b \geq 0$. If $\Phi \in \mathbf{C}_{a s s}(X, T)$ (resp. $\Phi \in \mathbf{C}_{a a}(X, T)$ ), then

(1) $\Psi$ and $\frac{a}{a+b} \Psi \in \mathbf{C}_{a s s}(X, T)$ (resp. $\Psi$ and $\frac{a}{a+b} \Psi \in \mathbf{C}_{a a}(X, T)$ );

(2) $P^{\mathbf{a}}(X, \Phi)=(a+b) P\left(Y, \frac{a}{a+b} \Psi\right)(P(Y, \Psi)$ is defined in (1.2));

(3) $\mu$ is an a-weighted equilibrium state of $\Phi$ iff $\nu=\mu \circ \pi^{-1}$ is an equilibrium state of $\frac{a}{a+b} \Psi$ and $\mu$ is a conditional equilibrium state of $\frac{1}{a} \Phi$ with respect to $\nu$, where $\frac{1}{a} \Phi=\left(\log \left(\phi_{n}^{\frac{1}{a}}\right)\right)_{n=1}^{\infty}$.

Letting $\mathbf{a}=(a, b) \in \mathbb{R}^{2}$ so that $a>0$ and $b \geq 0$, a measure $\mu \in \mathcal{M}(X, T)$ is called an a-weighted Gibbs measure if there exists $Q_{2}>0$ such that

$$
Q_{2}^{-1} \leq \frac{\mu([I]) \psi_{n}(\pi[I])^{\frac{b}{a+b}}}{\exp \left(-\frac{n}{a+b} P^{\mathbf{a}}(X, \Phi)\right) \phi_{n}(I)^{\frac{1}{a}}} \leq Q_{2}
$$

where

$$
\psi(J)=\sum_{I \in X_{n}: \pi I=J} \phi_{n}([I])^{\frac{1}{a}}, \forall J \in Y_{n} .
$$

The following theorem was also proved in [1. It shows that the a-weighted Gibbs measure exists uniquely for $\Phi \in \mathbf{C}_{a a}(X, T)$ with the bounded distortion property.

Theorem 2.5. Let $\pi: X \rightarrow Y$ be a one-block factor. Let $\mathbf{a}=(a, b) \in \mathbb{R}^{2}$ so that $a>0$ and $b \geq 0$. Let $\Phi \in \mathbf{C}_{a a}(X, T)$ and satisfy the bounded distortion property. Then

(1) $\Phi$ has a unique a-weighted equilibrium measure, say $\mu$;

(2) $\mu$ is also the unique $\mathbf{a}$-weighted Gibbs measure of $\Phi$; 
(3) if we define $\nu=\mu \circ \pi^{-1}$, then there exists $Q_{3}$ and $Q_{4}>0$ such that for all $J \in Y_{n}, n \in \mathbb{N}$ and $I=\pi(J)$,

$$
Q_{3}^{-1} \leq \frac{\nu([J])}{\exp \left(-\frac{n}{a+b} P^{\mathbf{a}}(X, \Phi)\right) \psi_{n}([J])^{\frac{a}{a+b}}} \leq Q_{3}
$$

and

$$
Q_{4}^{-1} \leq \frac{\mu([I])^{a} \nu(\pi[J])^{b}}{\exp \left(-n P^{\mathbf{a}}(X, \Phi)\right) \phi_{n}([I])} \leq Q_{4} .
$$

Combining Theorem 2.4 and Theorem 2.5 we have the following.

Proposition 2.6. Let $\Phi \in \mathbf{C}_{a a}(X, T)$ and satisfy the bounded distortion property and let $\nu \in \mathcal{M}(Y, S)$ be the Gibbs measure of $\Psi=\left(\log \psi_{n}\right)_{n=1}^{\infty}$ as defined in (2.22); it is thus an equilibrium measure of $\Psi$. Then $\Phi$ has a unique conditional equilibrium measure $\mu$ with respect to $\nu$ and there exists a constant $C_{4}>0$ such that

$$
C_{4}^{-1} \leq \frac{\mu([I]) \psi_{n}(\pi([I]))}{\nu(\pi([I])) \phi_{n}([I])} \leq C_{4} \forall I \in X_{n} \text { and } n \in \mathbb{N},
$$

where $\psi(J)=\sum_{I \in X_{n}: \pi(I)=J} \phi_{n}([I])$ and $\phi_{n}([I])$ is defined in (2.8) $)$.

Next we show that $P(X, \Phi)=P(Y, \Psi)$.

Proposition 2.7. Let $\pi: X \rightarrow Y$ be a one-block factor map and $\Phi \in \mathbf{C}_{a s s}(X, T)$. Let $\Psi \in \mathbf{C}_{a s s}(Y, S)$ be defined in (2.22). Then

$$
P(X, \Phi)=P(Y, \Psi) \text {. }
$$

Proof. Taking $\mathbf{a}=(1,0) \in \mathbb{R}^{2}$, it follows from (2.11) and Theorem 2.4 that

$$
\begin{aligned}
P^{\mathbf{a}}(X, \Phi) & =\sup \left\{\Phi_{*}(\eta)+h_{\eta}(T): \eta \in \mathcal{M}(X, T)\right\} \\
& =P(Y, \Psi) .
\end{aligned}
$$

Combining (2.20) and Theorem 2.2 with the fact that $\Phi \in \mathbf{C}_{a s s}(X, T)$ yields

$$
P(X, \Phi)=P^{\mathbf{a}}(X, \Phi)=\sup \left\{\Phi_{*}(\eta)+h_{\eta}(T): \eta \in \mathcal{M}(X, T)\right\}=P(Y, \Psi) .
$$

This completes the proof.

We end this subsection by introducing the relativised variational principle, which was developed by Ledrappier, Walters, Cao, Zhao, Feng and Huang (cf. [5], 8], 9] and [1]). This will be useful in the study of the relationship between $P(X, \Phi)$ and $P(Y, \pi \Phi)$.

Proposition 2.8 (Lemma 3.1 of [1). Let $\Phi \in \mathbf{C}_{a a}(X, T)$ and satisfy the bounded distortion property. If $\nu \in \mathcal{M}(Y, S)$, then:

(1) The relativised variational principle holds:

$$
\int_{Y} P\left(X, \Phi, \pi^{-1}(y)\right) d \nu(y)=\sup \left\{h_{\mu}(T)-h_{\nu}(S)+\Phi_{*}(\mu)\right\},
$$

where the supremum is taken over all $\mu \in \mathcal{M}(X, T)$ with $\mu \circ \pi^{-1}=\nu \in$ $\mathcal{M}(Y, S)$. 
(2) Define $\Psi=\left(\log \psi_{n}\right)_{n=1}^{\infty}$ on $Y$, where

$$
\psi_{n}(y)=\sum_{I \in X_{n}:[I] \cap \pi^{-1}(y) \neq \emptyset} \sup _{x \in[I] \cap \pi^{-1}(y)} \phi_{n}(x) .
$$

Then

$$
\begin{aligned}
\int_{Y} P\left(X, \Phi, \pi^{-1}(y)\right) d \nu(y) & =\sup \left\{h_{\mu}(T)-h_{\nu}(S)+\Phi_{*}(\mu)\right\} \\
& =\Psi_{*}(\nu),
\end{aligned}
$$

where the supremum is taken over all $\mu \in \mathcal{M}(X, T)$ with $\mu \circ \pi^{-1}=\nu \in$ $\mathcal{M}(Y, S)$ and $\Psi_{*}(\nu)$ is defined in (2.6).

\section{Proofs of Theorem A And Theorem B}

In this subsection we give the proofs of Theorem B and Theorem A. In the following, we assume $\Psi=\left(\log \psi_{n}\right)_{n=1}^{\infty}$, as defined in (2.22).

Proof of Theorem B. Take $\mathbf{a}=(1,0) \in \mathbb{R}^{2}$. It follows from Theorem 2.4 and Proposition 2.3 that we have $\pi \Phi, \Psi \in \mathbf{C}_{a a}(Y, S)$. Define the partition functions

$$
Z_{n}(X, \Phi)=\sum_{I \in X_{n}} \phi_{n}(I), Z_{n}(Y, \Psi)=\sum_{J \in Y_{n}} \psi_{n}(J)
$$

and

$$
Z_{n}(Y, \pi \Phi)=\sum_{J \in Y_{n}}(\pi \phi)_{n}(J)
$$

One can easily check that $\left(\log Z_{n}(X, \Phi)\right)_{n=1}^{\infty} \in \mathbf{C}_{a s s}(X, T)$, and $\left(\log Z_{n}(Y, \Psi)\right)_{n=1}^{\infty}$, $\left(\log Z_{n}(Y, \pi \Phi)\right)_{n=1}^{\infty} \in \mathbf{C}_{a s s}(Y, S)$. By the standard argument, we conclude that $P(X, \Phi), P(Y, \Psi)$ and $P(Y, \pi \Phi)$ exist. Since $P(X, \Phi)=P(Y, \Psi)$ (Proposition 2.7), for $\varepsilon>0$ there exists $N_{1} \in \mathbb{N}$ such that if $n \geq N_{1}$ we have

$$
\exp (-n \varepsilon) \leq \frac{Z_{n}(X, \Phi)}{Z_{n}(Y, \Psi)} \leq \exp (n \varepsilon)
$$

Let $\nu \in \mathcal{M}(Y, S)$ be the Gibbs measure of $\Psi$ as in Proposition 2.3 and $\mu \in \mathcal{M}(X, T)$ be its unique conditional equilibrium measure according to Proposition 2.6. Then there exist $Q_{3}$ and $Q_{4}>0$ such that for all $I \in X_{n}$ and $J=\pi I \in Y_{n}$ with $n \in \mathbb{N}$,

$$
Q_{3}^{-1} \exp (-n P(X, \Phi)) \phi_{n}([I]) \leq \mu([I]) \leq Q_{3} \exp (-n P(X, \Phi)) \phi_{n}([I])
$$

and

$$
Q_{4}^{-1} \exp (-n P(Y, \Psi)) \psi_{n}([J]) \leq \nu([J]) \leq Q_{4} \exp (-n P(Y, \Psi)) \psi_{n}([J]) .
$$

Since $\mu$ and $\nu$ are Gibbs on $X$ and $Y$, they are also ergodic. It follows from the Shannon-McMillian-Brieman Theorem, for $\varepsilon>0$ there exists $N_{2}>0$ such that if $n \geq N_{2}$ and $\mu$-a.e. $I \in X_{n}$ with $J=\pi([I]) \in Y_{n}$,

$$
\exp \left(-n\left(h_{\mu}(T)+\varepsilon\right)\right) \leq \mu([I]) \leq \exp \left(-n\left(h_{\mu}(T)-\varepsilon\right)\right)
$$

and

$$
\exp \left(-n\left(h_{\nu}(S)+\varepsilon\right)\right) \leq \nu([J]) \leq \exp \left(-n\left(h_{\nu}(S)-\varepsilon\right)\right) .
$$


Let $n \geq \max \left\{N_{1}, N_{2}\right\}$. Combining (3.3), (3.4), (3.5), (3.6) and (3.7) with the fact that $P(Y, \Psi)=P(X, \Phi)$ we have

$$
\begin{aligned}
& Z_{n}(X, \Phi) \leq Z_{n}(Y, \Psi) \exp (n \varepsilon) \\
&=\exp (n \varepsilon) \sum_{J \in Y_{n}} \psi_{n}([J])=\exp (n \varepsilon) \sum_{J \in Y_{n}} \psi_{n}([J]) \phi_{n}([I])^{-1}(\pi \phi)_{n}([J]) \\
& \leq Q_{3} Q_{4} \exp (n \varepsilon) \exp (n(P(Y, \Psi)-P(X, \Phi))) \sum_{J \in Y_{n}} \nu([J]) \mu^{-1}([I])(\pi \phi)_{n}([J]) \\
&(3.8) \quad \leq Q_{3} Q_{4} \exp \left(n\left(h_{\mu}(T)-h_{\nu}(S)+3 \varepsilon\right)\right) \sum_{J \in Y_{n}}(\pi \phi)_{n}([J]) .
\end{aligned}
$$

For the opposite inequality, we have

$$
\begin{aligned}
Z_{n}(X, \Phi) \geq & \exp (-n \varepsilon) Z_{n}(Y, \Psi)=\exp (-n \varepsilon) \sum_{J \in Y_{n}} \psi_{n}([J]) \phi_{n}([I])^{-1}(\pi \phi)_{n}([J]) \\
\geq & Q_{3}^{-1} Q_{4}^{-1} \exp (-n \varepsilon) \exp (n(P(Y, \Psi)-P(X, \Phi))) \\
& \quad \times \sum_{J \in Y_{n}} \nu([J]) \mu^{-1}([I])(\pi \phi)_{n}([J]) \\
\geq & Q_{3}^{-1} Q_{4}^{-1} \exp \left(n\left(h_{\mu}(T)-h_{\nu}(S)-3 \varepsilon\right)\right) \sum_{J \in Y_{n}}(\pi \phi)_{n}([J])
\end{aligned}
$$

Then (1.3) follows by dividing both sides of (3.8) and (3.9) with $n$ and taking $n$ to infinity. This completes the proof of Theorem B.

For the proof of Theorem A, we need the following results. They show that under the same assumption of Theorem A with the fact that $\pi$ has diamond, $P(X, \Phi)$ is strictly larger than $P(Y, \pi \Phi)$.

Theorem 3.1. Let $\pi: X \rightarrow Y$ be a one-block factor with diamond. Let $\Phi \in$ $\mathbf{C}_{a a}(X, T)$ and satisfy the bounded distortion property. If $\nu \in \mathcal{M}(Y, S)$ is the equilibrium state $\Psi=\left(\log \psi_{n}\right)_{n=1}^{\infty}$ as defined in (2.22) and $\mu$ is the conditional equilibrium state of $\Phi$ with respect to $\nu$, then

$$
h_{\mu}(T)>h_{\nu}(S) .
$$

Furthermore, $P(X, \Phi)>P(Y, \pi \Phi)$.

Proof. Since $\pi$ has diamond, then by Theorem 3.6 of 2 there exists $y \in Y, C_{5}>0$ and $C_{6}>1$ such that

$$
\#\left\{I \in X_{n}: I \cap \pi^{-1}(y) \neq \emptyset\right\} \geq C_{5} C_{6}^{n} .
$$

It follows from Proposition 2.6 that there exists $C_{4}>0$ such that $\forall I \in X_{n}$ and $J=\pi([I]) \in Y_{n}$,

$$
C_{4}^{-1} \frac{\phi_{n}([I])}{\psi_{n}([J])} \leq \frac{\mu([I])}{\nu([J])} \leq C_{4} \frac{\phi_{n}([I])}{\psi_{n}([J])}
$$


For $\mu$-a.e. $I \in X_{n}$ with $y \in J=\pi(I)$, it follows from (3.11), (3.12) and the Shannon-McMillian-Brieman Theorem that

$$
\begin{aligned}
h_{\mu}(T) & =\lim _{n \rightarrow \infty} \frac{-1}{n} \log \mu([I]) \\
& \left.\geq \lim _{n \rightarrow \infty} \frac{\log C_{4}}{n}+\lim _{n \rightarrow \infty} \frac{-1}{n} \log \left\{\frac{\phi_{n}([I])}{\psi_{n}([J])} \nu([J])\right\} \quad \text { (by (3.12) }\right) \\
& =\lim _{n \rightarrow \infty} \frac{-1}{n}\left[\log \frac{\phi_{n}([I])}{\psi_{n}([J])}\right]-\frac{1}{n} \log \nu([J]) \\
& =\lim _{n \rightarrow \infty} \frac{1}{n}\left[\log \sum_{I \in X_{n}: I \cap \pi^{-1}(y) \neq \emptyset} \phi_{n}([I])-\log \phi_{n}([I])\right]+\lim _{n \rightarrow \infty} \frac{-1}{n} \log \nu([J]) \\
& \geq \lim _{n \rightarrow \infty} \frac{1}{n}\left[\log C_{3}^{-1} C_{5} C_{6}^{n} \phi_{n}([I])-\log \phi_{n}(I)\right]+\lim _{n \rightarrow \infty} \frac{-1}{n} \log \nu([J]) \\
& =\log C_{6}+h_{\nu}(S) .
\end{aligned}
$$

The constant $C_{3}$ comes from the bounded distortion property for $\Phi$ (2.4) in Definition 2.1). Since $C_{6}>1$, we have $h_{\mu}(T)>h_{\nu}(S)$. Combining Theorem B and (3.10) we have $P(X, \Phi)>P(Y, \pi \Phi)$, and the proof is completed.

We continue the proof of Theorem A.

Proof of Theorem A. By the variational principle of $P(X, \Phi)$ and $P(Y, \pi \Phi)$ and Theorem 3.1, we only need to show that if $P(X, \Phi)>P(Y, \pi \Phi)$, then $\pi$ has diamond. Assume $\pi$ has no diamond. Then by Theorem 3.6 of [2], $h_{t o p}(X)=h_{\text {top }}(Y)$. Then $\pi: X \rightarrow Y$ is almost everywhere bounded-to-one. Using the identical argument of Theorem 3.1, we can also derive that $P(X, \Phi)=P(Y, \pi \Phi)$, a contradiction. This completes the proof of Theorem A

\section{Proof of Theorem C}

Let $\rho(\pi, \Phi)$ be as defined in (1.4). It follows from Theorem A that we have

Proposition 4.1. Under the same assumptions of Theorem A:

(1) If $\pi$ has no diamond, then $\rho(\pi, \Phi)=1$.

(2) If $\pi$ has diamond, then $\rho(\pi, \Phi)>1$.

We are ready to give the proof of Theorem C. Some auxiliary results are needed. First we define $X \backslash I=X \backslash \bigcup_{i \in \mathbb{N}} T^{-i}(I)$. The following result comes from [3].

Theorem 4.2 (Theorem 2.9 of [3]). Let $X$ be an SFT with positive topological entropy and let $\mu \in \mathcal{M}(X, T)$ be the measure of maximal entropy. Then for all $\varepsilon>0$ there exists $N \in \mathbb{N}$ such that if $n \geq N$ and $I \in X_{n}$, then

$$
h_{\mu}(X)-\varepsilon \leq h_{\mu}(X \backslash I) \leq h_{\mu}(X) .
$$

Remark 4.3. We remark here that in [3], Quas and Trow derived that

$$
h_{\text {top }}(X)-\varepsilon \leq h_{\text {top }}(X \backslash I) \leq h_{\text {top }}(X) .
$$

It is not hard to extend this result to (4.1) for $\mu$ is Gibbs for some potential $\Phi$ from their proof.

We now deduce the pressure from Theorem 4.2. 
Theorem 4.4. Let $X$ be a mixing SFT, and let $\Phi \in \mathbf{C}_{a a}(X, T)$ and satisfy the bounded distortion property. For $\varepsilon>0$, there exists an $N \in \mathbb{N}$ such that if $n \geq N$ and for all $I \in X_{n}$ where $X \backslash I$ is mixing, we have

$$
P(X, \Phi)-\varepsilon \leq P\left(X \backslash I,\left.\Phi\right|_{X \backslash I}\right)<P(X, \Phi) .
$$

Proof. Since $\Phi \in \mathbf{C}_{a a}(X, T)$ and satisfies the bounded distortion property, it follows from Proposition 2.3 that we may assume $\mu$ is the Gibbs measure for $\Phi$ on $X$. Let $\frac{\varepsilon}{2}>0$ and $I_{1} \in X_{n}$. According to the variational principle and Theorem 4.2.

$$
P(X, \Phi)-\Phi_{*}(\mu)-\varepsilon / 2=h_{\mu}(X)-\varepsilon / 2 \leq h_{\mu}\left(X \backslash I_{1}\right) .
$$

This shows that

$$
P(X, \Phi)-\varepsilon / 2 \leq h_{\mu}\left(X \backslash I_{1}\right)+\Phi_{*}(\mu) .
$$

We claim that $\Phi_{*}(\mu) \leq\left(\left.\Phi\right|_{X \backslash I_{1}}\right)_{*}(\mu)+\varepsilon / 2$. Indeed, since $\Phi \in \mathbf{C}_{a a}(X, T)$ we have

$$
\begin{aligned}
\Phi_{*}(\mu) & =\lim _{n \rightarrow \infty} \int_{X} \frac{1}{n} \log \phi_{n}(x) d \mu(x) \leq \int_{X} \frac{1}{n} \log \phi_{n}(x) d \mu(x) \\
& \leq \sum_{I \in X_{n}} \int_{I} \frac{1}{n} \log \phi_{n}(x) d \mu(x) \leq \sum_{I \in X_{n}} \frac{1}{n} \log \phi_{n}([I]) \mu([I]) \\
& \leq \sum_{I \in\left(X \backslash I_{1}\right)_{n}} \frac{1}{n} \log \phi_{n}([I]) \mu([I])+\frac{1}{n} \log \phi_{n}\left(\left[I_{1}\right]\right) \mu\left(\left[I_{1}\right]\right) \\
& \leq\left(\left.\Phi\right|_{X \backslash I_{1}}\right)_{*}(\mu)+\Phi_{*}(\mu) \mu\left(\left[I_{1}\right]\right)+\delta,
\end{aligned}
$$

for some small $\delta>0$. We note here that the $4^{\text {th }}$ inequality follows from the Birkhoff ergodic theorem and the fact that $\Phi \in \mathbf{C}_{a a}(X, T)$. Then the claim follows by taking $\mu\left(\left[I_{1}\right]\right)$ small enough and the fact that $\delta \rightarrow 0$ as $n \rightarrow \infty$. Therefore, it follows from (4.3) that we have

$$
\begin{aligned}
P(X, \Phi) & \leq h_{\mu}\left(X \backslash I_{1}\right)+\Phi_{*}(\mu)+\varepsilon / 2 \\
& \leq h_{\mu}\left(X \backslash I_{1}\right)+\left(\left.\Phi\right|_{X \backslash I_{1}}\right)_{*}(\mu)+\varepsilon . \\
& \leq P\left(X \backslash I_{1},\left.\Phi\right|_{X \backslash I_{1}}\right)+\varepsilon .
\end{aligned}
$$

This completes the proof.

Lemma 4.5. Under the same assumptions of Theorem A, Theorem $C$ holds if and only if for all $\varepsilon>0$ there exists a family of pairs $\left\{\left(\pi_{i}, X_{i}\right)\right\}_{i=1}^{k}$ such that

(1) $X_{i}$ is a subsystem of $X, \forall i=1, \cdots, k$,

(2) $X_{i}$ is an $S F T, \forall i=1, \cdots, k$, and

(3) $\left\{B\left(P\left(X_{i},\left.\Phi\right|_{X_{i}}\right), \varepsilon\right)\right\}_{i=1}^{k}$ forms an $\varepsilon$-cover of $[P(Y, \pi \Phi), P(X, \Phi)]$.

Proof. Let $\rho \in[1, \rho(\pi, \Phi)]$ and let $P(Y, \pi \Phi)=p>0$. For $\varepsilon p>0$ we assume that there exists a family of pairs $\left\{\left(\pi_{i}, X_{i}\right)\right\}_{i=1}^{k}$ where $\pi_{i}: X_{i} \rightarrow Y$ is a factor map and $X_{i}$ is an SFT $\forall i=1, \cdots, k$, and $\left\{B\left(P\left(T_{i},\left.\Phi\right|_{X_{i}}\right), \varepsilon p\right)\right\}_{i=1}^{k}$ forms an $\varepsilon p$-cover of $[P(Y, \pi \Phi), P(X, \Phi)]$. Since $\rho \in[1, \rho(\pi, \Phi)]$ and

$$
P(Y, \pi \Phi)=p \leq \rho p \leq \rho(\pi, \Phi) P(Y, \pi \Phi)=P(X, \Phi),
$$

i.e., $\rho p \in[P(Y, \pi \Phi), P(X, \Phi)]$, there exists an $1 \leq i \leq k$ such that $\rho p \in$ $B\left(P\left(X_{i},\left.\Phi\right|_{X_{i}}\right), \varepsilon p\right)$, i.e.,

$$
\left|\rho p-P\left(T_{i},\left.\Phi\right|_{X_{i}}\right)\right| \leq \varepsilon p .
$$


This means that $\left|\rho-\rho\left(\pi_{i},\left.\Phi\right|_{X_{i}}\right)\right| \leq \varepsilon$. On the other hand, for $\varepsilon>0$, take a sequence $1 \leq \rho_{1}=1, \rho_{2}, \cdots, \rho_{k-1}, \rho_{k}=\rho(\pi, \Phi) \leq \rho(\pi, \Phi)$ with

$$
\frac{\varepsilon}{2 p} \leq\left|\rho_{i+1}-\rho_{i}\right| \leq \frac{\varepsilon}{p} \text { for all } i \in 1, \cdots, k-1
$$

Define $I_{i}=\left[\rho_{i}, \rho_{i+1}\right]$ for $i \in 1, \cdots, k-1$. It follows from Theorem $\mathrm{C}$ that there exists a sequence $\left\{\left(\pi_{i}, X_{i}\right)\right\}_{i=1}^{k}$ where $\pi_{i}$ is a factor from $X_{i}$ to $Y, X_{i} \subseteq X$ is an SFT and $\rho\left(\pi_{i},\left.\Phi\right|_{X_{i}}\right) \in\left[\rho_{i}, \rho_{i+1}\right]$. If $i=k, P\left(X_{k},\left.\Phi\right|_{X_{k}}\right) \leq \rho_{k} P(Y, \pi \Phi)=P(T, \Phi)$, and if $i=1, P\left(X_{1},\left.\Phi\right|_{X_{1}}\right) \geq \rho_{1} P(Y, \pi \Phi)=P(Y, \pi \Phi)$. If $P(Y, \pi \Phi) \leq q \leq P(X, \Phi)$, then $1 \leq \frac{q}{p} \leq \rho(\pi, \Phi)$; thus $\frac{q}{p} \in I_{i}$ for some $i$. Therefore

$$
\left|\frac{q}{p}-\rho\left(\pi_{i}, X_{i}\right)\right| \leq \varepsilon .
$$

This implies that $\left|q-P\left(X_{i},\left.\Phi\right|_{X_{i}}\right)\right| \leq p \varepsilon$, and this means that $\left\{B\left(P\left(X_{i},\left.\Phi\right|_{X_{i}}\right)\right.\right.$, $p \varepsilon)\}_{i=1}^{k}$ forms a $p \varepsilon$-cover of $[P(Y, \pi \Phi), P(X \Phi)]$. This completes the proof.

Lemma 4.6. Let $\pi: X \rightarrow Y$ be a one-block factor with diamond. Then properties (1), (2) and (3) of Lemma 4.5 hold.

Proof. Without loss of generality we assume that $X$ is full shift. Since $\Phi \in$ $\mathbf{C}_{a a}(X, T)$, we conclude that $Z_{n}(X, \Phi)=\sum_{I \in X_{n}} \phi_{n}([I])$ is sub-additive. Then we have

$$
P(X, \Phi) \leq \frac{1}{n} \log Z_{n}(X, \Phi), \forall n \in \mathbb{N},
$$

and for $\varepsilon>0$ there exists $N_{1} \in \mathbb{N}$ such that if $n \geq N_{1}$, then

$$
P(X, \Phi) \geq \frac{1}{n} \log Z_{n}(X, \Phi)-\varepsilon .
$$

By Theorem 4.4 we choose $N_{2} \in \mathbb{N}$ such that if $n \geq N_{2}$ and for all $I \in X_{n}, X \backslash I$ is mixing, we have

$$
P(X, \Phi)-\varepsilon \leq P\left(X \backslash I,\left.\Phi\right|_{X \backslash I}\right)<P(X, \Phi) .
$$

Since $\pi: X \rightarrow Y$ has diamond, then for all $J \in Y_{n}$ with $n \in \mathbb{N}$,

$$
\#\left\{I \in X_{n}: \pi(I)=J\right\} \geq 1 \text {. }
$$

We define $S_{J}=\left\{I \in X_{n}: \pi(I)=J\right\}$ and arrange $\left\{J: J \in Y_{n}\right\}$ in the lexicographic order with $J_{1}<J_{2}<\cdots<J_{m}$ and define $S_{1}=S_{J_{1}}, S_{2}=S_{J_{2}}, \cdots, S_{m}=S_{J_{m}}$. We also arrange $S_{i}$ in the lexicographic order, i.e.,

$$
S_{i}=\left\{I_{j}^{(i)}\right\}_{j=1}^{\left|S_{i}\right|}, \text { for } i=1, \ldots, m,
$$

where $|A|$ denotes the number of elements of $A$. For all $i$, define

$$
\hat{S}_{i}=S_{i} \backslash I_{1}^{(i)}
$$

i.e., drop the first pattern in $S_{i}$ for all $i=1, \cdots, m$. Therefore $\left|\hat{S}_{i}\right|=\left|S_{i}\right|-1$ for $i=1, \ldots, m$. Letting $\left|\hat{S}_{1}\right|+\left|\hat{S}_{2}\right|+\cdots+\left|\hat{S}_{m}\right|=r(m)$, we put all elements of 
$\hat{S}_{1}, \cdots, \hat{S}_{m}$ together in order and renumber all its elements by $\left\{I_{j}\right\}_{j=1}^{r(m)}$, i.e.,

$$
\begin{aligned}
\left\{\hat{S}_{1}, \cdots, \hat{S}_{m}\right\} & =\left\{\left\{I_{2}^{(1)}, \cdots, I_{\left|\hat{S}_{1}\right|}^{(1)}\right\}, \cdots,\left\{I_{2}^{(1)}, \cdots, I_{\left|\hat{S}_{1}\right|}^{(1)}\right\}\right\} \\
& =\left\{I_{1}, I_{2}, \cdots, I_{r(m)}\right\} .
\end{aligned}
$$

Define $S=\left\{I_{1}, I_{2}, \cdots, I_{r(m)}\right\}$. We construct a family of subsystems of $X$ as follows:

(1) Let $X_{0}=X$ and $X_{1}=X \backslash I_{1}$.

(2) $X_{j}=X_{j-1} \backslash I_{j}$ for all $1 \leq j \leq r(m)$.

(3) Finally, $X_{r(m)}=X_{0} \backslash \bigcup_{j=1}^{r(m)} I_{j}$.

For $1 \leq j \leq r(m)$, define $\pi_{i}=\left.\pi\right|_{X_{i}}: X_{i} \rightarrow Y$. According to the construction, it can be easily checked that $\pi_{i}$ is a factor for all $i \in[1, r(m)]$, and it follows from Theorem 4.4 that

$$
P(X, \Phi)>P\left(X_{1},\left.\Phi\right|_{X_{1}}\right)>\cdots>P\left(X_{r(m)},\left.\Phi\right|_{X_{r(m)}}\right)
$$

and

$$
P\left(X_{i+1},\left.\Phi\right|_{X_{i+1}}\right) \geq P\left(X_{i},\left.\Phi\right|_{X_{i}}\right)-\varepsilon \text { for all } i \in[1, r(m)-1] .
$$

Finally, we claim that

$$
P\left(X_{r(m)},\left.\Phi\right|_{X_{r(m)}}\right) \leq P(Y, \pi \Phi)+\varepsilon .
$$

Indeed, it follows from (4.6) and Proposition 2.7 that

$$
P(Y, \Psi)=P(X, \Phi) \geq \frac{1}{n} \log Z_{n}(X, \Phi)-\varepsilon .
$$

Since

$$
Z_{n}(Y, \Psi)=\sum_{J \in Y_{n}} \sum_{I: \pi(I)=J} \phi_{n}(I)
$$

is also sub-additive, there exists $N_{3} \in \mathbb{N}$ such that if $n \geq N_{3}$, then

$$
P(Y, \Psi) \geq \frac{1}{n} \log Z_{n}(Y, \Psi)-\varepsilon .
$$

By the construction of $X_{r(m)}$, we have

$$
Z_{n}(Y, \Psi)=Z_{n}(Y, \pi \Phi)=Z_{n}\left(X_{r(m)}, \Phi\right),
$$

Combining (4.11), (4.5) and (4.12) we have that if $n \geq \max \left\{N_{1}, N_{2}, N_{3}\right\}$, then

$$
\begin{aligned}
P(Y, \pi \Phi) & \geq \frac{1}{n} \log Z_{n}(Y, \pi \Phi)-\varepsilon=\frac{1}{n} \log Z_{n}(Y, \Psi)-\varepsilon \\
& =\frac{1}{n} \log Z_{n}\left(X_{r(m)},\left.\Phi\right|_{X_{r(m)}}\right)-\varepsilon \geq P\left(X_{r(m)},\left.\Phi\right|_{X_{r(m)}}\right)-\varepsilon .
\end{aligned}
$$

Thus (4.9) holds, and it follows from (4.7), (4.8) and (4.9) that

$$
\bigcup_{i=1}^{r(m)} B\left(P\left(X_{i},\left.\Phi\right|_{X_{i}}\right), \varepsilon\right)
$$

forms an $\varepsilon$-cover of $[P(Y, \pi \Phi), P(X, \Phi)]$. The proof is completed.

Finally, we finish the proof of Theorem C.

Proof of Theorem $C$. The proof is obtained by combining Lemma 4.5 and Lemma 4.6. The proof is completed. 


\section{ACKNOWLedGement}

The authors would like to thank the referees for their suggestions, which led to a great improvement of this paper.

\section{REFERENCES}

[1] J. Barral and D. J. Feng, Weighted thermodynamic formalism and applications (2009), arXiv:0909.4247v1.

[2] R. Meester and J. Steif, Higher-dimensional subshifts of finite type, factor maps and measures of maximal entropy, Pacific J. Math. 2 (2001), 497-510. MR.1868699 (2002j:37028)

[3] A. Quas and P. Trow, Subshifts of multi-dimensional shifts of finite type, Ergodic Theory Dynam. Systems 20 (2000), 859-874. MR 1764932(2001d:37011)

[4] B. Kitchen, Symbolic dynamics, one-sided, two-sided and countable state Markov shifts. Springer-Verlag (1998). MR:1484730 (98k:58079)

[5] F. Ledrappier and P. Walters, A relativised variational principle for continuous transformations, J. London Math. Soc. 16 (1977), 568-576. MR0476995 (57:16540)

[6] P. Walters, An introduction to ergodic theory. Springer-Verlag (1982). MR648108 (84e:28017)

[7] Y. Zhao and Y. L. Cao, On the topological pressure of random bundle transformations in sub-additive case, J. Math. Anal. Appl. 342 (2008), 715-725. MR2440833 (2010d:37060)

[8] Y. L. Cao, D. J. Feng and W. Huang, The thermodynamic formalism for sub-additive potentials, Discrete Contin. Dyn. Syst. 20 (2008), 639-657. MR2373208 (2008k:37072)

[9] D. J. Feng and W. Huang, Lyapunov spectrum of asymptotically sub-additive potentials, Comm. Math. Phys. 297 (2010), 1-43. MR2645746

Department of Mathematics, National Dong Hwa University, Hualien 970003, Taiwan

E-mail address: jcban@mail.ndhu.edu.tw

Department of Mathematics, National Central University, Taoyuan 32001, Taiwan

E-mail address: chchang@mx.math.ncu.edu.tw 